\title{
HST/WFPC2 Photometry in the 30 Doradus Nebula Beyond R136
}

\author{
Rodolfo H. Barbá and Nolan R. Walborn \\ STScI, 3700 San Martin Dr., Baltimore, MD 21218, USA
}

\begin{abstract}
A photometric study of the 30 Doradus Nebula beyond the core is being carried out by means of HST/WFPC2 archival images, with special attention to the numerous multiple systems and compact clusterings found there.
\end{abstract}

30 Doradus is the nearest and hence best resolved extragalactic starburst. Knowledge of its stellar content is vital to the interpretation of more distant starbursts, as well as to fundamental astrophysical problems such as the IMF, stellar mass limit, stellar evolution, and the structure of giant $\mathrm{H}$ II regions. Recent studies of the stellar content of 30 Doradus with HST/WFPC2 and FOS have concentrated on the central cluster core, R136 (Hunter et al. 1995 and references therein; Massey \& Hunter 1998).

However, R136 and its immediate surroundings account for only a third to a half of the ionization of 30 Dor. Other very massive stars and stellar systems are distributed throughout the several-arcminute extent of the Nebula. They include objects both older and younger than R136; there is evidence that the formation of the latter has been triggered by the energetic activity of R136. So far, these important surrounding populations have been investigated only with groundbased observations (Parker 1993; Walborn \& Blades 1997). In the latter spectral-classification study, five spatially and/or temporally distinct stellar components were isolated within the Nebula. But numerous multiple systems remain unresolved in these populations, particularly in the younger ones.

On the basis of HST/WFPC2 archival images (Proposals 5114, 5589, and 6122 ), a photometric study of the 30 Doradus Nebula surrounding R136 is being carried out, with special emphasis on the numerous multiple systems and compact clusterings found there. Of particular interest are systems in the bright nebular filaments where current massive-star formation is taking place, as revealed by both groundbased and HST/NICMOS infrared images (see Walborn \& Barbá and Barbá et al. in these proceedings). Special attention is given to the objects included in the above groundbased spectral-classification study. Magnitudes and colors are derived for the newly resolved components of the multiple systems, while their ages and evolutionary status will be inferred insofar as possible. However, it is well known that the effective temperatures and masses of hot stars are degenerate when derived from photometry alone. Hence, this project is viewed as preparation for followup spatially resolved spectroscopy with HST/STIS, in order to advance our knowledge of the entire stellar content of 30 Doradus to the current state of the art, as is warranted by its unique status. 

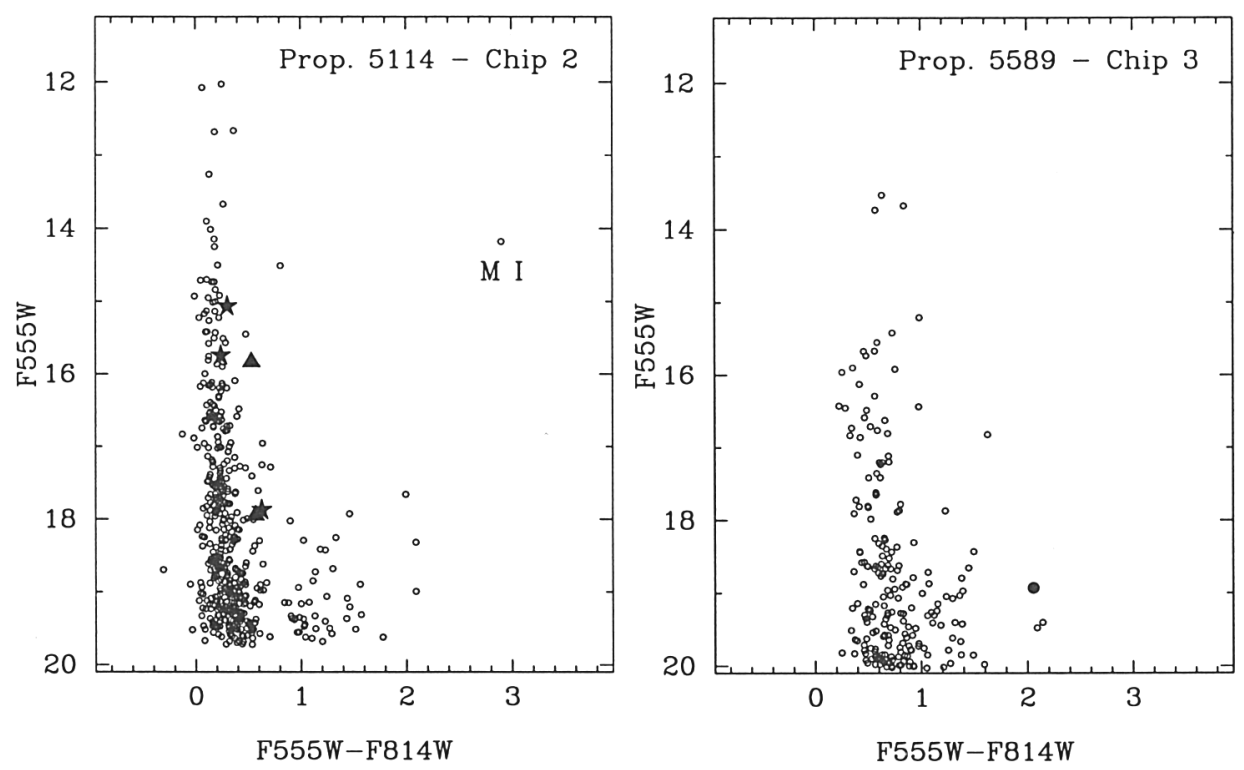

Figure 1. HST/WFPC2 color-magnitude diagrams of two areas of the 30 Doradus Nebula surrounding R136.

As an example, CMDs of two areas are shown in Fig 1 . On the left is a CMD corresponding to the area E-NE of R136, where the young multiple systems Knots 1 and 2 are located. On the right is a CMD of the area S-SW. The comparison of these CMDs reveals clear differences between areas located at the same distance from R136: in the $\mathrm{NE}$ a main sequence is well defined, but in contrast the SW area shows a less sharp main sequence, perhaps due to higher reddening, pre-main-sequence objects, and/or fewer very high-mass objects. The M I star in the NE is likely an unrelated LMC field star in projection. Stars and triangles in the left CMD mark the Knots 1 and 2 stellar components, respectively. Note the magnitude and color similarities between the two Knots, in accordance with their spectroscopic morphologies. The black circle in the right CMD is a new IR source discovered $1^{\prime}$ south of R136 in HST/NICMOS images by Walborn et al. 1999 .

Acknowledgments. Support for this work was provided by NASA through grants numbers GO-7819.01-96A and AR-7545.01-96A from STScI, which is operated by AURA, Inc., under NASA contract NAS 5-26555.

\section{References}

Hunter, D.A., et al. 1995, ApJ, 448, 179

Massey, P., \& Hunter, D.A. 1998, ApJ, 493, 180

Parker, J.Wm. 1993, AJ, 106, 560

Walborn, N.R., et al. 1999, AJ, 117, 225

Walborn, N.R., \& Blades, J.C. 1997, ApJS, 112, 457 